\title{
ACTITUDES LINGÜÍSTICAS HACIA EL PROFESORADO DE INGLÉS NATIVO Y NO NATIVO ${ }^{1}$
}

\author{
LINGUISTIC ATTITUDES TOWARDS NATIVE AND NON-NATIVE ENGLISH \\ TEACHERS
}

\section{ATITUDES LINGÜÍSTICAS PARA COM PROFESSORES NATIVOS E NÃO NATIVOS DE INGLÊS}

Iván Martínez Rivas ${ }^{1}$ 0000-0003-1182-1106

José Luis Estrada Chichón² 0000-0003-3274-6133

${ }^{1}$ Universidad Alfonso X El Sabio - Madri, Espanha; irivamar@myuax.com

${ }^{2}$ Universidad de Cádiz - Cádiz, Espanha; joseluis.estrada@uca.es

\section{RESUMO:}

O interesse pelo fator falante nativo no ensino da língua inglesa é recente, sendo o trabalho de Medgyes (1992) o ponto de partida. Vários estudos trataram das preferências de estudantes e professores, embora nenhum estudo tenha investigado esta variável na Galiza (Espanha) e, mais especificamente, no Bacharelado (16-18 anos de idade). Portanto, este artigo visa contribuir para a literatura específica existente, analisando as atitudes e preferências dos estudantes $(n=101)$ e professores $(n=6)$ em relação aos professores de inglês nativos e não nativos em dois anos de Bachillerato e em duas escolas diferentes na Galiza. Os dados foram obtidos por meio de um questionário (LASAGABASTER; SIERRA, 2005) e dois grupos focais com parte dos alunos $(n=12)$ e professores $(n=6)$ pesquisados, respectivamente, combinando métodos quantitativos e qualitativos. Os resultados revelam uma tendência geral em favor dos professores nativos de inglês que se torna mais forte à medida que os alunos avançam nas etapas educacionais: educação primária, secundária e terciária. Além disso, em termos de preferência por áreas linguísticas, os professores não nativos são preferidos para ensinar gramática e questões de avaliação, sendo superados pelos professores nativos em todas as outras áreas.

Palavras chave: atitude do estudante; atitude do professor; ensino de idiomas.

\begin{abstract}
:
Interest in the field of the native-speaker factor in English teaching is recent, the starting point being the work by Medgyes (1992). Several studies have explored learner and teacher preferences, although no study has investigated this variable in Galicia (Spain) and, more specifically, at the Bachillerato educational stage (16-18 years). Therefore, this research paper aims to contribute to the existing specific literature by analyzing the attitudes and preferences of students $(n=101)$ and teachers $(n=6)$ towards native and non-native teachers of English in the two Years of Bachillerato and two different secondary schools in Galicia. Data were obtained using a questionnaire (LASAGABASTER; SIERRA, 2005) and two focus groups along with a representative sample of students $(n=12)$ and teachers $(n=6)$, including quantitative and qualitative research methods. The results show a general trend in favor of native teachers of English which becomes more significant as students progress through the educational stages: primary, secondary, and tertiary education. In terms of preference for language category, non-

\footnotetext{
${ }^{1}$ El presente artículo es el resultado de un Trabajo de Fin de Máster (TFM) presentado en una universidad de España en el curso académico 2020-21 como requisito para la obtención del Máster en Formación del Profesorado de Educación Secundaria Obligatoria y Bachillerato, Formación Profesional y Enseñanzas de Idiomas.
} 
native teachers are preferred for teaching grammar and dealing with assessment issues, while native teachers are preferred for all other categories.

Keywords: student attitudes; teacher attitudes; language instruction.

\section{RESUMEN:}

El interés por el factor nativo en la enseñanza del inglés es reciente, siendo el trabajo de Medgyes (1992) el punto de partida. Diversas investigaciones han tratado las preferencias de alumnos y docentes, aunque ningún estudio ha investigado esta variable en Galicia (España), y, más específicamente, en Bachillerato (16-18 años). Por ello, este artículo pretende contribuir a la literatura específica existente, analizando las actitudes y preferencias de estudiantes $(n=101)$ y docentes $(n=6)$ ante profesores de inglés nativos y no nativos en dos cursos de Bachillerato y en dos centros educativos distintos de Galicia. Los datos se obtuvieron mediante un cuestionario (LASAGABASTER; SIERRA, 2005) y dos grupos focales con una parte de los estudiantes $(n=12)$ y docentes $(n=6)$ encuestados, respectivamente, combinando métodos cuantitativos y cualitativos. Los resultados revelan una tendencia general a favor de los profesores de inglés nativos que va afianzándose según los alumnos progresan por las etapas educativas: educación primaria, secundaria y terciaria. Además, en cuanto a la preferencia de las áreas lingüísticas, se opta por el profesorado no-nativo para la enseñanza de la gramática y cuestiones de evaluación, siendo superado por el profesorado nativo en el resto de las áreas.

Palabras clave: actitud del estudiante; actitud del docente; enseñanza de idiomas

\section{Introducción}

Desde la publicación del artículo pionero de Medgyes (1992) sobre profesores de lengua extranjera nativos y no nativos, el interés en este ámbito de investigación ha ido en aumento. Algunos estudios se han centrado en actitudes del profesorado (p. ej.: LLURDA; HUGUET, 2003; REVES; MEDGYES, 1994), mientras que otros han investigado las diferentes creencias desde el punto de vista del estudiantado (p. ej.: LASAGABASTER; SIERRA, 2005; TSOU; CHEN, 2017). Sin embargo, ningún trabajo se ha centrado en la investigación de las creencias y preferencias de ambos estudiantes y docentes ante Profesores Nativos de Inglés (PNI en adelante) y Profesores No Nativos de Inglés (PNNI en adelante) en la etapa educativa de Bachillerato (16-18 años) en el contexto específico de la comunidad autónoma de Galicia, España.

En las últimas décadas, con la expansión del inglés y su emergencia como lengua franca (JENKINS, 2009; LLURDA; BAYYURT; SIFAKIS, 2017), el aprendizaje del inglés como lengua extranjera (ILE en adelante) ha ido cobrando más y más relevancia. Asimismo, este creciente interés por el ILE puede estar relacionado con la promoción del plurilingüismo por parte de la Unión Europea, y, por consiguiente, arraigando el inglés en el sistema educativo e incrementando la popularidad de colegios plurilingües (CALAFATO, 2019; PÉREZ VIDAL, 2011). No obstante, en la práctica docente, se puede observar todavía dificultades de aprendizaje del ILE (EDUCATION FIRST, 2020). En ocasiones se pretenden mitigar estos 
déficits con la contratación de PNI, sirviendo estos como 'modelos lingüísticos'. A pesar de ello, la incorporación de PNI no tiene por qué verse necesariamente reflejado en la correcta adquisición del ILE por parte del alumnado (LLURDA, 2018). Por tanto, en el presente artículo se pretende ahondar en el estudio de esa dicotomía, comprobando las diferencias actitudinales en el alumnado y profesorado de Bachillerato entre PNI y PNNI en un contexto de análisis concreto con el objeto de observar sus implicaciones para el aula de ILE.

\section{La enseñanza del inglés como lengua extranjera en Bachillerato}

Durante el último siglo, el inglés se ha expandido debido a las diferentes situaciones geopolíticas y socioeconómicas, y, a última instancia, a la globalización (MANSFIELD; POPPI, 2012). Esto dio lugar a una nueva jerarquía lingüística mundial, con el inglés como lengua global debido a su uso para propósitos internacionales (DOR̈NYEI; CSIZER; NEMETH, 2006). Datos de Ethnologue (EBERHARD; SIMONS; FENNIG, 2021) revelan que el inglés es la lengua más hablada del mundo con más de 1.500 millones de hablantes, de los cuales solo 375 millones son nativos. Como resultado, han aparecido distintas realizaciones del idioma en forma de variedades lingüísticas (KACHRU, 1965). Con todo, académicos como Jenkins (2009; 2015), por ejemplo, diferencian ILE de inglés como lengua franca (ILF en adelante). Mientras que el ILE es dependiente de la norma e implica tener como modelo la gramática y pronunciación de Reino Unido o Estados Unidos, el ILF emerge de hablantes no nativos, donde la comunicación no implica necesariamente la adecuación a la norma (JENKINS, 2015). Siendo así, los hablantes de ILF buscan comunicarse, incluso utilizando el acento de su lengua materna (L1 en adelante), sin recurrir a la pronunciación de las variedades modelo nativas de inglés, al contrario del caso de la enseñanza del ILE.

Respecto a la distinción nativo frente a no-nativo, Moussu y Llurda (2008) anuncian que estas etiquetas deben tomarse con especial cautela, ya que resulta difícil categorizar a individuos concretos. Un ejemplo es el estudio de Brutt-Griffler y Samimy (2001), donde los participantes tenían problemas para denominarse nativos o no nativos debido a su contexto personal. De modo que el concepto de hablante nativo puede definirse desde diversas perspectivas: desde usuario de L1 (DEWAELE, 2018) o como un constructo social basado en la nacionalidad sin base biológica (HOLLIDAY, 2013). A efectos de una mayor claridad sobre esta dicotomía, en el presente estudio se utilizará la etiqueta de nativo para referirse a docentes nacidos y criados en países pertenecientes al círculo interno (Reino Unido, Estados Unidos, Canadá, Australia, Nueva Zelanda y Sudáfrica) (KACHRU, 1985) y no nativo a profesores de 
países donde se hablan variedades del inglés, ya que estos términos están reconocidos ampliamente en este campo de estudio (LASAGABASTER; SIERRA, 2005; ALONSOHERRERO Y LASAGABASTER, 2019).

Con todo, incluso ante el aumento en España de la promoción de la lengua inglesa en la escuela (VILLACAÑAS, 2017), el nivel de inglés parece estancado y superado por otros países europeos (EDUCATION FIRST, 2020). Debido a esto, y ante el creciente interés por el factor nativo como 'modelo lingüístico', muchos colegios optan por la contratación de profesorado nativo, preferiblemente como asistentes de conversación. Es por esto por lo que cabe tener en cuenta una vía de colaboración entre docentes nativos y no nativos. La colaboración docente de ambos perfiles lingüísticos genera un incremento motivacional en el alumnado (CARLESS; WALKER, 2006). Sin embargo, esta colaboración ha probado ser compleja, ya que normalmente son los PNNI los docentes principales o titulares, teniendo que cubrir un currículo muy amplio, mientras que los PNI funcionan como auxiliares de conversación, repercutiendo en una falta de tiempo para cubrir la programación didáctica esperada, entre otros.

De cara a analizar el rol docente de cada uno de ellos colaborando en equipo, los PNI se centran normalmente en el uso lingüístico del ILE, tratando errores y sugiriendo alternativas, mientras que los PNNI demuestran y diagnostican el porqué de esos errores (SU; SHANG, 2020). Esto puede ser a causa de lo que Andrews (2007) explica como la diferencias entre el conocimiento de la lengua y el conocimiento sobre la lengua, siendo los PNNI habitualmente más competentes en lo último y los PNI en lo primero (LLURDA; BAYYURT; SIFAKIS, 2017). Cabe también decir que la colaboración docente en una misma aula puede ser un arma de doble filo para el alumnado, ya que se incrementa la interacción profesorprofesor en perjuicio de pensamiento activo de los alumnos, que pasan a ser meros espectadores (SU, 2021).

\section{Profesores de inglés nativos y no nativos}

La figura del PNNI ha estado vinculada a la enseñanza de lenguas, ya que tradicionalmente esta ha estado en manos de profesores que compartían la misma L1 que los alumnos (LLURDA, 2018). Sin embargo, hay que esperar hasta finales del siglo XX, con la publicación del trabajo de Medgyes (1992) poniendo en cuestión la relevancia de los profesores nativos, y confirmado a posteriori por Braine (1999), cuando se abre el debate sobre profesores nativos frente a profesores no nativos. El de Reves y Medgyes (1994) fue el primer 
estudio que comparó las características de los PNI y PNNI. Se identificaron características de enseñanza de ambos tipos de docentes mediante cuestionarios. Los resultados mostraron que los PNI se caracterizaban por tener una mejor pronunciación y soltura, mientras que los PNNI fueron definidos con un mayor conocimiento en la lengua y una mejor gramática, pero dependientes del libro de texto. Estos mismos resultados fueron confirmados posteriormente por Llurda (2005).

Por otra parte, se puede observar una discriminación hacia los PNNI que se materializa en la falta de acceso a puestos de trabajos, restringiéndolos a los PNI (SELVI, 2010). Esto se debe a una conjetura indiscutida del docente nativo como profesor ideal y, como consecuencia, relegando al profesorado no-nativo a un estatus inferior (LLURDA, 2018). De modo que contribuir aportando datos positivos acerca de los PNNI es de vital importancia para transformar las identidades de los docentes no nativos (LLURDA, 2018). Llurda, Bayyurt y Sifakis (2017) manifiestan que mientras los PNNI han pasado un proceso de formación lingüística en la segunda lengua (L2 en adelante), algunos PNI carecen de esa formación en su L1 y muchas veces son designados a posiciones docentes por el mero hecho de ser nativos. Asimismo, muchos PNI son monolingües y no han pasado por el proceso de aprendizaje de una L2, a diferencia de sus homólogos no nativos.

Los estudiantes, en sus preferencias ante la opción PNI o PNNI, son más propensos a elegir a los PNI (TODD; POJANAPUNYA, 2009), sobre todo si alguna vez asistieron a clases de uno (MOUSSU; LLURDA, 2008). No obstante, algunos estudios como los de Lasagabaster y Sierra (2005) o Benke y Medgyes (2005), por ejemplo, concluyen que para tareas determinadas y en áreas concretas, los alumnos llegan a mostrar preferencia hacia los PNNI sobre los PNI (MOUSSU; LLURDA, 2008). En el siguiente apartado se clarificará el concepto de actitudes lingüísticas y sus implicaciones para el estudio del factor nativo en docentes de ILE.

\section{Actitudes lingüísticas}

El concepto de actitud lingüística ha sido la piedra angular de la psicología social tradicional (EDWARDS, 1994), siendo el tema central de multitud de estudios sociolingüísticos en los últimos 50 años. Sin embargo, es un concepto difícil de definir, ya que en muchas ocasiones se solapa con otros conceptos diferentes en el campo de la psicología social, como pueden ser creencias, opiniones, hábitos o ideologías (MCKENZIE, 2010). Al definir el término, se pueden encontrar varias versiones, aportando cada una diferentes 
perspectivas a la conceptualización del este. Eagly y Chaiken (1993) definen las actitudes como "una tendencia psicológica que se expresa evaluando una entidad particular con cierto grado de aprobación o desaprobación” (p. 1). Más tarde, Garret (2010) define las actitudes como una evaluación hacia un objeto social de cualquier tipo. Así, las actitudes lingüísticas podrían ser definidas como reacciones a una lengua concreta. Por consiguiente, tienen un rol de vital importancia en el aprendizaje de idiomas, pudiendo llegar incluso a determinar el éxito o el fracaso del alumnado (ALONSO-HERRERO; LASAGABASTER, 2019; DOR̈NYEI; CSIZER; NEMETH, 2006; GARRET, 2010). Por otra parte, los juicios o evaluaciones que se hacen sobre las lenguas dan lugar a la categorización del objeto como correcto o incorrecto, capturando las convenciones sociales en las respectivas comunidades y desvelando el estatus de prestigio o desprestigio (O'ROURKE, 2011). Por tanto, con el estudio actitudinal, se pueden observar e inferir los prejuicios que existen hacia una lengua o grupo concreto; en el caso de esta investigación, el factor nativo en docentes de ILE.

En línea con lo anterior, según Baker (1992), el estudio de las actitudes lingüísticas se constituye de tres componentes básicos: primero, el cognitivo, siendo este el conjunto de ideas y creencias; segundo, el afectivo, esto es, los sentimientos hacia algo concreto; y, tercero, el conductual, que apunta directamente a la materialización de acciones específicas. El componente conductual suele ser diferencial a la hora de tener en cuenta las actitudes hacia los PNI y PNNI, ya que las tendencias suelen variar en términos de comportamiento, tal y como es en el caso de las prácticas docentes y la proficiencia idiomática (ZHANG, 2016). Con esto en mente, la investigación actitudinal se articula principalmente a la luz de dos paradigmas sociales: el conductista, que percibe las actitudes como respuestas evidentes y observables a situaciones sociales; y el cognitivista, en el cual las actitudes son un estado de preparación mental inferidas con el estímulo correcto (O’ROURKE, 2011). Con todo, numerosos estudios han desacreditado la visión conductista, ya que solo tiene en cuenta las actitudes como única variable que influye en la conducta, dejando de lado otros factores como la edad, género o contexto lingüístico, que pueden intervenir e influenciar altamente la conducta (MCKENZIE, 2010).

\section{Objetivos de investigación}

Por todo lo expuesto, el presente trabajo busca dar respuesta a la siguiente pregunta de investigación: ¿cuáles son las actitudes linguiísticas del alumnado y profesorado de Bachillerato hacia los PNI y PNNI en el contexto de análisis? Esta pregunta sirve para orientar 
la formulación del objetivo de investigación: explorar las actitudes del alumnado y profesorado de Bachillerato ante los PNI y PNNI y sus implicaciones en el aula de ILE. De este objetivo de investigación principal se desprenden tres objetivos específicos:

1. Identificar si el alumnado muestra una mayor preferencia hacia los PNI o PNNI.

2. Determinar cuáles son las preferencias del alumnado y profesorado hacia los PNI o PNNI según áreas lingüísticas específicas.

3. Explorar las implicaciones didácticas extraídas de los resultados respecto a la distinción PNI-PNNI.

\section{Metodología}

La presente investigación tiene un alcance de tipo transversal, ya que se recogen datos en un solo momento en el tiempo, y se caracteriza por tener un enfoque mixto, ya que se obtienen datos cuantitativos de una encuesta actitudinal y cualitativos de un grupo focal, tal y como se explica más adelante. Por una parte, los datos cuantitativos fueron obtenidos mediante un cuestionario (Lasagabaster y Sierra, 2005) sobre actitudes lingüísticas hacia profesores nativos y no nativos de idiomas. Por otra parte, se llevaron a cabo tres grupos focales, dos de ellos con alumnos $(n=12)$ y otro con PNI y PNNI $(n=6)$, una vez codificados y analizados los datos de las encuestas. Las variables para tener en consideración son la preferencia del alumnado hacia PNI o PNNI; la etapa educativa predilecta de los estudiantes para asistir a clase con PNI o PNNI (educación primaria, educación secundaria y educación terciaria); y las áreas lingüísticas donde los PNI y PNNI tienen una mayor influencia: gramática, vocabulario, pronunciación, listening, speaking, reading, writing, estrategias de aprendizaje, cultura, actitudes y evaluación.

La muestra total de este estudio consiste en 101 estudiantes (51 hombres y 50 mujeres) de $1^{\circ}$ y $2^{\circ}$ de Bachillerato (16-18 años), que residen en la localidad de Ribiera, Galicia, España. En este contexto se hablan mayoritariamente dos lenguas, gallego y español, siendo ambas lenguas oficiales en el territorio. Mientras que la lengua más hablada y mayoritaria es el español, el gallego es una lengua minoritaria y minorizada (O'ROURKE, 2011). Por su parte, el inglés funciona como la principal lengua extranjera en Galicia, incluida como asignatura en el currículo escolar. Por otro lado, los participantes estaban divididos en cuatro clases distribuidas en dos centros educativos distintos. Ambos colegios tenían un profesor titular nonativo y un auxiliar de conversación nativo que impartía docencia junto al profesor no-nativo una hora por semana en cada curso. La población objeto de estudio son los estudiantes de 
Bachillerato que hayan sido expuestos al inglés nativo y no-nativo y que tengan o hayan tenido un PNI y PNNI en educación reglada. Cabe señalar que se prestó la atención adecuada en cuanto a los procedimientos éticos mediante consentimientos informados. El director de cada centro educativo firmó una copia del formulario de consentimiento informado, se envió una copia a cada familia y cada participante aceptó ser parte del estudio.

La primera encuesta que se administró fue el cuestionario actitudinal sobre PNI y PNNI dirigido al alumnado seleccionado de Lasagabaster y Sierra (2005). Este cuestionario se divide en dos partes: información general y cuestionario actitudinal. La primera sección sirve para recoger información sobre el contexto de la muestra, incluyendo preguntas de tipo personal. La única modificación registrada respecto al cuestionario original supuso el cambio del idioma "euskera" por el "gallego" en las preguntas 5 y 6, ya que al tratarse de un contexto diferente, la lengua cooficial no sería el euskera sino el gallego. Además, se tradujo al español por estar el cuestionario original escrito en inglés. La segunda sección incluye preguntas actitudinales sobre PNI y PNNI. La razón por elegir el cuestionario de Lasagabaster y Sierra (2005) es que se diseñó para un contexto multilingüe (País Vasco) pero que comparte múltiples similitudes con el contexto de la presente investigación (Galicia) por lo expuesto arriba.

El cuestionario consiste en 42 afirmaciones en las que los estudiantes fueron preguntados por su opinión sobre si estaban más interesados en PNI o PNNI, en relación con diferentes categorías: las cuatro destrezas lingüísticas, gramática, vocabulario, estrategias de aprendizaje, cultura y civilización y evaluación y estas con relación a las tres principales etapas educativas: educación primaria, secundaria y terciaria. Las posibles respuestas consistían en una escala Likert de 5 puntos entre 1 (muy en descuerdo) y 5 (muy de acuerdo).

Segundo, el método directo de recogida de datos consistió en un grupo focal desarrollado para compilar datos cualitativos. Como explica Llurda (2018), apoyar cuestionarios actitudinales con el uso de entrevistas o grupos focales ayuda a tener una imagen más clara de los resultados. Los grupos focales se llevaron a cabo utilizando elementos estructurados y algunos no estructurados para así disminuir la ansiedad de los participantes (GIBSON; HUA, 2016), donde el entrevistador y uno de los autores del presente trabajo funciona como facilitador en una conversación guiada, exponiendo los temas y haciendo preguntas si el tema se desviaba. Las peguntas principales fueron construidas a partir de los resultados obtenidos en los cuestionarios, siendo validadas por un experto en la enseñanza del ILE. Los participantes de los grupos focales fueron 12 alumnos, divididos en dos grupos, uno por cada centro educativo. Por otra parte, también se entrevistó a sus profesores $(n=6)$, nativos 
y no nativos: ¿Cuáles son los pros y contras de tener un PNI y un PNNI?; según los resultados de la encuesta, más de la mitad de los alumnos $(52,5 \%)$ prefieren a un PNI, mientras que a un $36,6 \%$ les da igual y solo un 10,9\% prefiere a un PNNI, ¿a qué cree que se debe esto?; por otra parte, un 64,3\% preferiría tener a ambos profesores. ¿Encuentra alguna razón?; según los resultados de la encuesta, existe un incremento en la preferencia hacia los PNI según se va progresando por de las diferentes etapas educativas, mientras que en educación primaria el porcentaje es de un 56,5\%, en educación secundaria es un 66,4\% y en educación terciaria, un 67,4\%. ¿A qué cree que puede deberse esto?; en cuanto a la enseñanza de la pronunciación y el vocabulario, solo un 5\% y 8\%, respectivamente, prefiere a un PNI, mientras que en materia de speaking los porcentajes están más repartidos, ¿por qué cree que sucede esto?; en términos de evaluación ¿cree que un profesor nativo es mejor evaluando? Los resultados dicen que para todas las destrezas se prefiere a un PNNI. El valor más alto para el PNNI es $41 \%$ (pronunciación) y el más bajo, 20\% (writing); cuanto más alto es su nivel de competencia en inglés, más aprecia al PNI, ¿está de acuerdo?; y, ¿hay alguna cuestión relacionada con los profesores nativos o no nativos que no hayamos tratado y de la que le gustaría hablar?

Los datos cuantitativos fueron obtenidos mediante un cuestionario online en la plataforma Google Forms. Primero, se contactó con los colegios y, después de obtener el consentimiento de la Dirección, se enviaron consentimientos informados a las familias. Una vez aceptada la participación en el proyecto, se realizó en la semana del 19 de abril de 2021 en dos centros educativos de Ribeira. Aunque la toma de datos se realizó mediante una plataforma virtual, se decidió que hiciesen la encuesta todos a la vez en horas lectivas para evitar el efecto bola de nieve y tener el máximo control sobre la muestra, ya que según Johnstone (2016), debido al carácter online de recogida de datos, existe el riesgo de que los participantes reenvíen el cuestionario o no sean ellos quien lo cumplimentan. Para hacerles llegar el cuestionario, se creó un blog en la plataforma Blogspot con una entrada que incluía el link de la encuesta.

Una vez codificados y analizados los datos de las encuestas, se llevaron a cabo tres grupos focales, dos de ellos con alumnos y otro con profesores nativos y no nativos de manera online mediante la plataforma Zoom. En las entrevistas a los alumnos, podían utilizar tanto gallego como español, según estuviesen más cómodos, siendo ambas sus lenguas nativas. Sin embargo, en el caso del grupo focal con los profesores, se decidió utilizar el inglés como lengua vehicular debido a la baja competencia lingüística de los PNI en gallego y español.

Los datos obtenidos mediante los instrumentos anteriormente presentados se 
analizarán de una manera cuantitativa y cualitativa debido a la naturaleza mixta del estudio. Para los datos cuantitativos, una vez codificados en Excel los datos del cuestionario, se empleará el software SPSS Statistics para llevar a cabo un análisis descriptivo por porcentajes de la muestra. Debido a las variables elegidas para el estudio, no se ha decido hacer un análisis inferencial, centrándose el análisis en uno de carácter descriptivo. Por otra parte, en cuanto a los datos cualitativos, las respuestas de los grupos focales recogidas en las grabaciones serán codificadas mediante etiquetas de información para su posterior análisis descriptivo y poder así enriquecer y dar consistencia a los datos cuantitativos obtenidos del cuestionario.

\section{Resultados}

El grupo de participantes que cubrieron el cuestionario actitudinal fueron 101 estudiantes de Bachillerato, de los cuales un $40,6 \%$ son de $1^{\circ}$ curso y un $59,4 \%$ de $2^{\circ}$ curso. En cuanto a la especialidad, un 40,0\% pertenecen a Ciencias Sociales y Humanidades, mientras que el $60,0 \%$ restante es de la especialidad de Ciencias. Sobre la edad de los informantes, esta oscila entre los 16 y 18 años $(\mathrm{M}=16,93 ; \mathrm{DS}=.73)$ y en relación con el género, un $50,5 \%$ se etiquetaron como hombres y un 49,5\% como mujeres. Ante la pregunta de cuál es su L1, casi la mitad de los participantes (49,5\%) eligió el español, mientras que un 26,6\% manifestó ser bilingüe (español y gallego), seguido por solo un 23,8\% que declaró ser monolingüe en gallego.

Sobre la autopercepción de la competencia idiomática de los participantes en las diferentes lenguas con las que están en contacto, un 47,6\% reporta tener un nivel alto de gallego, seguido de cerca por los niveles medio (46,5\%) y bajo (5,9\%). En cuanto al español, una gran mayoría $(80,2 \%)$ señala un nivel alto, mientras que un 19,8\% declara tener un nivel medio. En relación con el inglés, casi la mitad de los informantes $(47,5 \%)$ indica un nivel medio, en contraposición con el 31,7\% que revela un nivel bajo y un 20,8\% un nivel alto. Respecto a otras lenguas, una gran mayoría $(72,3 \%)$ habla de un nivel bajo, mientras que el $26,7 \%$ reporta un nivel medio y solo un $1,0 \%$ se posiciona en un nivel alto.

Por último, en cuanto a la exposición al inglés nativo, el total los encuestados declaró haber tenido al menos una vez un profesor de ILE nativo, aunque solo un tercio de ellos $(36,7 \%)$ señaló haber estado alguna vez en un país de habla inglesa. Asimismo, los participantes revelaron que llevan estudiando inglés una media de 10,6 años en educación reglada entre educación primaria y secundaria, incluyendo aquí Bachillerato.

Sobre el grupo focal dirigido al alumnado, participaron 12 estudiantes que fueron 
divididos en dos grupos, uno por cada centro educativo. De entre todos los participantes (mujeres: $58,0 \%$; y hombres: $42,0 \%$ ), un $42,0 \%$ son alumnos de $1^{\circ}$ de Bachillerato y el 58,0\% restante son alumnos de $2^{\circ}$ de Bachillerato, con una media de edad de 17,1 años (DS=.79). Por otra parte, se llevó a cabo un grupo focal con 6 profesores (PNNI: 66,0\%; y PNI: 34,0\%), que imparten docencia en los dos centros educativos. Mientras que los PNNI son profesores titulares del centro, los PNI son auxiliares de conversación británicos que solamente permanecen en el centro educativo durante un curso académico.

Los datos obtenidos del cuestionario actitudinal sobre PNI y PNNI se analizan según la variable de preferencia de los alumnos hacia un tipo de docente u otro, la etapa educativa y las áreas de enseñanza específicas, respectivamente mediante un análisis descriptivo por porcentajes. De cara a analizar los datos incluidos en las tablas que se presentan a continuación de una manera más clara, se combinan los porcentajes de las categorías muy en desacuerdo (1) y en desacuerdo (2), haciendo referencia a una preferencia hacia PNNI, y también se unirán las categorías de acuerdo (4) y muy de acuerdo (5), mostrando una tendencia hacia PNI, con el fin de obtener una mejor presentación de los resultados. Por último, la categoría ni de acuerdo ni en desacuerdo (3) permanecerá igual, mostrando una tendencia central de indiferencia ante PNI o PNNI.

La primera variable de investigación que se va a examinar es la preferencia general de los alumnos de Bachillerato sobre tipo de docente según el factor nativo. Más de la mitad de la muestra $(52,5 \%)$ tiene una preferencia hacia PNI, mientras que un $36.6 \%$ no tiene una preferencia clara, y solo un 10,9\% de los participantes elegiría un PNNI antes que un PNI. La tendencia general muestra una clara inclinación hacia los PNI. Sin embargo, el porcentaje de informantes que no muestran una clara preferencia también es relativamente alto $(36,6 \%)$ :

Tabla 1 - "En general, preferiría a un profesor nativo"

\begin{tabular}{lll}
\hline & $\mathrm{n}$ & $\%$ \\
\hline 1: muy en desacuerdo & 4 & 4,0 \\
2: en desacuerdo & 7 & 6,9 \\
3: ni de acuerdo ni en desacuerdo & 37 & 36,6 \\
4: de acuerdo & 20 & 19,8 \\
5: muy de acuerdo & 33 & 32,7 \\
\hline
\end{tabular}

Fuente: elaboración propia

Respecto al segundo objeto de estudio, las tendencias del alumnado en materia de elección de un tipo de docente según la etapa educativa, se puede observar un incremento en la preferencia hacia PNI mientras se avanza en las respectivas etapas educativas. Para educación primaria (6-12 años), un 56,5\% prefiere un PNI, porcentaje que se incrementa para 
educación secundaria (12-18 años) (66,4\%) y llegando hasta el 67,4\% para educación terciaria (18-). En contraposición con los datos de preferencia hacia PNNI, que caen drásticamente con un $10,9 \%$ de preferencia en educación primaria, $9,9 \%$ en educación secundaria y 8,0\% en educación terciaria. A la hora de reconocer la posibilidad de tener a ambos profesores en una enseñanza colaborativa, un 45,5\% está muy de acuerdo, en contraposición con el 5,0\% que se muestra muy en desacuerdo. Por tanto, se puede observar una clara tendencia a tener ambos profesores $(64,3 \%)$, por encima de la preferencia hacia PNI $(52,5 \%)$ :

Tabla 2 - "Preferiría un profesor nativo en educación primaria, secundaria, terciaria o ambos profesores"

\begin{tabular}{lrrrrc}
\hline & 1 & 2 & 3 & 4 & 5 \\
\hline PNI en educación primaria & $7,9 \%$ & $8,9 \%$ & $26,7 \%$ & $12,9 \%$ & $43,6 \%$ \\
PNI en educación secundaria & $3,0 \%$ & $6,9 \%$ & $23,8 \%$ & $33,7 \%$ & $32,7 \%$ \\
PNI en educación terciaria & $4,0 \%$ & $4,0 \%$ & $24,8 \%$ & $22,8 \%$ & $44,6 \%$ \\
Ambos profesores & $5,0 \%$ & $5,9 \%$ & $24,8 \%$ & $18,8 \%$ & $45,5 \%$ \\
\hline
\end{tabular}

Fuente: elaboración propia

La tercera variable consiste en examinar las diferentes tendencias de los alumnos en cuanto al factor nativo en diferentes áreas de enseñanza. Cabe decir que las categorías listening, speaking, reading y writing recogidas en el cuestionario hacen referencia a los cuatro bloques (compresión de textos orales, producción de textos orales, compresión de textos escritos y producción de textos escritos) incluidos en el Decreto 86/2015, por el que se establece el currículo de la Educación Secundaria Obligatoria y del Bachillerato en la Comunidad Autónoma de Galicia. Para la mejor compresión de los alumnos a la hora de cumplimentar el cuestionario, se ha decidido usar esta categorización según destrezas lingüísticas, debido a su estandarización en la clase de ILE. Los resultados describen un sesgo claro hacia los PNI en las áreas de vocabulario $(84,1 \%)$ y pronunciación $(82,2 \%)$, con más de tres cuartos de la muestra a favor, en contraposición con los valores hacia PNNI (5,0\% y 8,0\%, respectivamente), que caen drásticamente. En otras áreas, la preferencia hacia PNI desciende ligeramente hasta el 65,3\% para la categoría de actitudes, el 63,3\% para reading $(63,3 \%)$ y un 61,4\% en relación con actitudes positivas de aprendizaje del ILE. Estos valores están claramente en oposición con el 10,9\%, 15,8\% y 8,9\% de alumnos que elegirían un PNNI en lugar de un PNI en estas áreas.

Por otra parte, se muestra una preferencia hacia los PNI en áreas de speaking (48,5\%), listening $(45,5 \%)$ y estrategias de aprendizaje (54,5\%), en contraste con un $28,7 \%, 26,7 \%$ y $12,9 \%$ de tendencia a favor de los PNNI, porcentaje relativamente más alto que en áreas de 
vocabulario $(5,0 \%)$ y pronunciación $(8,0 \%)$. Sobre el aprendizaje de elementos culturales de países de habla inglesa, un 40,6\% prefiere un PNI, seguido de cerca por un 32,7\% que elegiría un PNNI y, por último, un $26,7 \%$ no tiene una preferencia. Por último, en cuanto a la gramática, esta es la única categoría en la que los participantes prefieren a un PNNI $(41,6 \%)$ sobre un PNI (23,8\%). No obstante, un 34,0\% no muestra una preferencia clara:

Tabla 3 - "Un profesor nativo es mejor en ciertas áreas, en general"

\begin{tabular}{llllll}
\hline & \multicolumn{1}{c}{1} & \multicolumn{1}{c}{2} & \multicolumn{1}{c}{3} & \multicolumn{1}{c}{4} & \multicolumn{1}{c}{5} \\
\hline Gramática & $14,9 \%$ & $26,7 \%$ & $34,7 \%$ & $14,9 \%$ & $8,9 \%$ \\
Vocabulario & $4,0 \%$ & $1,0 \%$ & $10,9 \%$ & $15,8 \%$ & $68,3 \%$ \\
Pronunciación & $5,0 \%$ & $3,0 \%$ & $9,9 \%$ & $13,9 \%$ & $68,3 \%$ \\
Listening & $18,8 \%$ & $7,9 \%$ & $27,7 \%$ & $26,7 \%$ & $18,8 \%$ \\
Reading & $6,9 \%$ & $2,0 \%$ & $27,7 \%$ & $27,7 \%$ & $35,6 \%$ \\
Speaking & $15,8 \%$ & $12,9 \%$ & $22,8 \%$ & $18,8 \%$ & $29,7 \%$ \\
Writing & $14,9 \%$ & $15,8 \%$ & $49,5 \%$ & $12.9 \%$ & $6,9 \%$ \\
Estrategias de aprendizaje & $5,0 \%$ & $7,9 \%$ & $32,7 \%$ & $20,8 \%$ & $33,7 \%$ \\
Cultura y civilización & $24,8 \%$ & $7,9 \%$ & $26,7 \%$ & $23,8 \%$ & $16,8 \%$ \\
Actitudes países habla inglesa & $8,9 \%$ & $6,9 \%$ & $18,8 \%$ & $15,8 \%$ & $49,5 \%$ \\
Actitudes aprender inglés & $5,9 \%$ & $5,0 \%$ & $27,7 \%$ & $17,8 \%$ & $43,6 \%$ \\
\hline
\end{tabular}

Fuente: elaboración propia

En cuanto a la preferencia en términos de evaluación, no existe una tendencia clara hacia ninguno de los dos tipos de docentes para writing $(49,5 \%)$, reading $(42,6 \%)$, speaking $(46,6 \%)$ y gramática $(39,7 \%)$. Asimismo, cabe destacar una ligera preferencia hacia los PNNI, ya que respecto al writing, un 30,7\% prefiere un PNNI sobre un PNI (19,8\%). Esta tendencia permanece en reading (32,7\% y 23,7\%, respectivamente), speaking (39,6\% y 23,7\%, respectivamente) y gramática $(34,7 \%$ y $25,8 \%$, respectivamente). Sobre la pronunciación y listening, se muestra una tendencia favorable hacia los PNNI con un 38,6\% y 37,6\%, respectivamente, seguido de cerca por "ni de acuerdo ni en desacuerdo" con un 32,7\% para ambas categorías y, por último, el PNI con unos valores inferiores de 29,7\% en listening y $28,8 \%$ en términos de pronunciación:

Tabla 4 - "Un profesor nativo me evaluaría mejor en ciertas áreas"

\begin{tabular}{lrrrrr}
\hline & 1 & 2 & 3 & 4 & \multicolumn{1}{c}{5} \\
\hline Listening & $18,8 \%$ & $18,8 \%$ & $32,7 \%$ & $10,9 \%$ & $18,8 \%$ \\
Reading & $13,9 \%$ & $19,8 \%$ & $42,6 \%$ & $5,9 \%$ & $17,8 \%$ \\
Speaking & $22,8 \%$ & $16,8 \%$ & $42,6 \%$ & $5,9 \%$ & $17,8 \%$ \\
Writing & $14,9 \%$ & $15,8 \%$ & $49,5 \%$ & $12,9 \%$ & $6,9 \%$ \\
Pronunciación & $16,8 \%$ & $21,8 \%$ & $32,7 \%$ & $13,9 \%$ & $14,9 \%$ \\
Gramática & $12,9 \%$ & $21,8 \%$ & $39,6 \%$ & $13,9 \%$ & $11,9 \%$ \\
\hline
\end{tabular}

Fuente: elaboración propia 
Debido a que esta investigación es de naturaleza mixta, se recogieron también datos cualitativos para triangular la información y así garantizar la validez del estudio. Las respuestas de los participantes del grupo focal fueron categorizadas para su posterior interpretación. Para llevar a cabo dicha categorización, se determinó el número de temas citados frecuentemente por los informantes y se crearon etiquetas de información para presentar los datos de una manera más ilustrativa (para garantizar el anonimato de los participantes, de cara a presentar sus comunicaciones personales, estas se representarán mediante códigos: "AL" para los alumnos; y "PNI" y "PNNI" para los docentes, seguido del número de participante). A continuación, se descubren las categorías extraídas de las respuestas de los grupos focales; primero, se interpretan las respuestas del grupo focal dirigido a los alumnos y luego se analiza la información relativa al grupo focal dirigido a los docentes según áreas temáticas:

- Alumnos. Al mostrar los pros y contras de tener un PNI, la muestra analizada opina que al PNI cuesta entenderlo, pero practicas más inglés con él y las clases son más divertidas. En cuanto al PNNI, consideran que sabe mucha gramática, pero manda muchos deberes. Igualmente, un PNNI enseña mejor, aunque usa mucho el libro, las clases son más aburridas y no pronuncia bien. Sobre la competencia idiomática, un PNI habla más fluido, tiene más nivel y sabe más vocabulario que un PNNI. Sin embargo, el acento español hace al PNNI más inteligible, ya que se entiende mejor. Asimismo, también se pone en valor el acento nativo como modelo lingüístico. Con todo, son bastante críticos hacia el sistema educativo y ponen en duda la figura del PNI. Sobre la comunicación en el aula, ponen en valor la L1 del PNNI, valorando los beneficios de tener un profesor bilingüe, mientras que con un PNI la comunicación es más difícil. Para ellos también tiene valor el proceso de aprendizaje de la LE por parte del PNNI. En materia de preferencia según el nivel educativo, consideran que, a más nivel, el PNI es más necesario, poniendo de manifiesto la importancia de explicaciones en español, la L1 de los alumnos, sobre todo en niveles bajos, y valorando al PNNI para etapas inferiores. En cuanto a la autopercepción de dominio idiomático, consideran que cuanto más nivel más necesitas un PNI y que las actitudes positivas hacia los PNI se incrementan según el nivel idiomático. Respecto a la posibilidad de la enseñanza colaborativa, muestran un rechazo al profesor nativo, y en cuanto a su experiencia con ambos docentes en el aula, siguen manifestando su descontento, mientras también muestran la dependencia del PNI 
con el PNNI. Por último, teniendo presentes las áreas lingüísticas, indican que necesitan un PNI en speaking y pronunciación, mientras que la mayoría considera que un PNNI es indispensable para explicar gramática. Sobre la evaluación, creen que un PNNI sabe hacerlo de una manera más correcta, lo que no les beneficia en términos de calificación.

- Docentes. Los PNNI destacan la importancia de la formación lingüística en la LE, aunque muestran que los PNI son más competentes en términos de léxico y pronunciación. Por otra parte, los PNI son conscientes de su déficit lingüístico en español. Se ponen de manifiesto prejuicios, ya que consideran que los PNNI tienen un estatus inferior a los PNI. También destacan problemas de cara a acceder a puestos de trabajo y a la promoción por parte de sectores educacionales. De la misma forma, abogan por una enseñanza colaborativa, en la que los PNI enseñarían mayormente pronunciación, clases de conversación y léxico, y los PNNI se centrarían en la enseñanza de gramática. En último lugar, ilustran la importancia de la formación en componente didáctico y lingüístico, es decir, la necesidad de que ambos profesores se formen, ya que un docente no es tal por el mero hecho de ser nativo en un idioma.

\section{Discusión}

El presente trabajo explora las preferencias generales de los alumnos y docentes de Bachillerato en cuanto al factor nativo en la enseñanza de inglés en relación con la distinción PNI y PNNI; las tendencias de los alumnos hacia los PNI o PNNI según la etapa educativa (educación primaria, secundaria y terciaria); y sus diferentes preferencias hacia PNI o PNNI según el área lingüística. Así, teniendo en cuenta la primera variable de análisis, se puede observar una tendencia general del alumnado a favor de los PNI, en línea con estudios previos como Lasagabaster y Sierra (2005), Rámila (2015) y Todd y Pojanapunya (2009). Sin embargo, incluyendo en la ecuación la docencia colaborativa, los datos cuantitativos muestran una preferencia hacia tener ambos profesores (FAUZI; HASHIM, 2020; RÁMILA, 2015; TSOU; CHEN, 2017; WALKINSHAW; OAHN, 2014), incluso llegando a situarse por delante de los PNI, al igual que en Lasagabaster y Sierra (2005).

No obstante, estos datos son rebatidos por la representación de alumnos que participaron en el grupo focal, mostrando un rechazo a los PNI, para quienes se muestran dependientes de los PNNI: "es bueno tener un profesor no nativo y nativo a la vez, pero un 
nativo no sería capaz de dar la clase sin el no-nativo" (AL3, comunicación personal, 10 de mayo de 2021), percibiéndolo más como un complemento al PNI que como un docente per se, tal y como concluye Su (2021), ilustrando la enseñanza colaborativa como compleja e incluso contraproducente. También muestran su rechazo a los PNI en la enseñanza colaborativa en contraste con Carless y Walker (2006), interpretando sus propuestas didácticas como poco funcionales: "el nativo no nos viene a enseñar nada, viene solo a hablar, que para eso podíamos tener un video de YouTube" (AL3, comunicación personal, 10 de mayo de 2021) y "lo único bueno que tiene un PNI son las explicaciones de fonética, por lo demás es una pérdida de tiempo" (AL12, comunicación personal, 10 de mayo de 2021). Esto puede deberse a que los participantes caracterizan a los PNI como poco dependientes del libro de texto a diferencia de sus homólogos no nativos, como cercioran Reves y Medgyes (1994) y Llurda (2005). Por tanto, como resultado de las actividades comunicativas y lúdicas que plantean los PNI, al no utilizar el libro de texto, los alumnos creen que no están aprendiendo nada, debido a la idea equivocada del libro como base para el aprendizaje, arraigada en el sistema educativo tradicional (GARCÍA MATA, 2008).

En el grupo focal, los alumnos también hacen referencia a lo que Andrews (2007) llama el conocimiento sobre la lengua que tienen los PNNI: "cuando están los dos en clase, y hay que explicar algo de gramática, lo explica siempre el de aquí porque es el que sabe" (AL1, comunicación personal, 10 de mayo de 2021). Sin embargo, en cuanto a las respuestas de los docentes, se muestran a favor de la docencia colaborativa, mediante una repartición de tareas: "para enseñar conversación es mejor un PNI, cuando hacemos clases colaborativas, intento que se centre en vocabulario y fonética y yo doy la gramática cuando doy la clase solo" (PNNI2, comunicación personal, 12 de mayo de 2021), pero también indican la necesidad de formación lingüística y pedagógica de los PNI, tal como apuntan Llurda, Bayyurt y Sifakis (2017), ya que muchas veces para los PNI no es requisito para dar clase debido a su condición de nativos: "es igual de importante el componente didáctico como el lingüístico, necesitas también formación en didáctica y en atención a la diversidad, y muchas veces los nativos no tienen esa formación ya que no suele ser un requisito para ellos" (PNNI3, comunicación personal, 12 de mayo de 2021).

En segundo lugar, en lo referido a las preferencias según la etapa educativa, los resultados del cuestionario sugieren una tendencia del alumnado hacia PNI, incrementándose a medida que se sube el nivel educativo, corroborado por los datos cualitativos: "cuándo subes de nivel, también necesitas un profesor con más nivel. En primaria necesito que me expliquen 
en castellano, pero en la universidad, como es más nivel, necesito un profesor nativo" (AL11, comunicación personal, 10 de mayo de 2021), en concordancia con Lasagabaster y Sierra (2002; 2005), y Madrid y Pérez Cañado (2004). Por otra parte, en cuanto a la proficiencia de los docentes de ILE, los datos de los grupos focales posicionan a los PNI como docentes con un mayor conocimiento, por eso son necesarios para niveles educativos más altos, en línea con los resultados de Reves y Medgyes (1994). No obstante, también son críticos con el sistema educativo y diferencian entre la enseñanza formal y la no reglada para la preferencia docente, poniendo en duda la figura del PNI en Bachillerato: "yo lo que quiero es aprobar la EBAU [(examen de acceso a educación terciaria)], y para eso el nativo no me vale (AL2, comunicación personal, 10 de mayo de 2021)" y “tú no tienes que aprender inglés, sino que tienes que aprender cómo aprobar el examen, no es lo mismo Bachillerato que una academia de idiomas" (AL11, comunicación personal, 10 de mayo de 2021).

Los resultados del grupo focal muestran que los alumnos tienen una autoconsciencia de nivel en inglés baja, conectándolo con la inteligibilidad del acento español en la LE: "necesito esa entonación española porque no tengo mucho nivel y al nativo no lo entiendo bien" (AL3, comunicación personal, 10 de mayo de 2021), tal y como se concluye en el estudio de Dewaele y Pena (2018). No obstante, como explica García Mata (2008), esta necesidad de explicación y clarificación en la L1 de los estudiantes puede estar relacionada con las malas prácticas docentes en clase de ILE por parte de los PNNI, utilizando demasiado su L1. Asimismo, se pone en valor el hecho de que el PNNI sea bilingüe y conocedor de la L1 de los estudiantes: "yo prefiero a un profesor no nativo porque necesito que a veces me expliquen las cosas en español" (AL4, comunicación personal, 10 de mayo de 2021), favoreciendo la comunicación en el aula como se explica en Benke y Medgyes (2005) y Tsou y Chen (2017). Los alumnos también mencionan la importancia del proceso de aprendizaje de la LE que tuvieron los PNNI a diferencia de los PNI: "un profesor español te lo va a explicar de una forma que lo entiendas porque ya estudió inglés y te puede aconsejar" (AL12, comunicación personal, 10 de mayo de 2021) y "sabe los trucos y aprendes más rápido" (AL10, comunicación personal, 10 de mayo de 2021). Por otro lado, en el grupo focal dirigido a profesores, se ponen de manifiesto prejuicios hacia los PNNI y dificultades para conseguir trabajo, restringiéndolos a los PNI (SELVI, 2010) y considerando a los PNNI con un estatus inferior (Llurda, 2018): "creo que los alumnos prefieren nativos porque promocionan mucho a los PNI en las academias" (PNI2, comunicación personal, 12 de mayo de 2021) y "a mí me ha pasado que en algunos trabajos han elegido a nativos antes que a mí por el mero hecho de 
ser nativo" (PNNI1, comunicación personal, 12 de mayo de 2021).

Por último, la tercera variable del estudio explora la preferencia en áreas específicas. Los profesores fueron mejor valorados en el área de gramática en sintonía con estudios cualitativos como Lai Ping (2012) y Walkinshaw y Oahn (2014), pero en contraste con los resultados de Fauzi y Hashim (2020). Asimismo, estos resultados corroboran estudios cuantitativos previos como Rámila (2015), Árva y Medgyes (2000) y Lasagabaster y Sierra (2005). Los resultados del grupo focal dirigido a profesores revelan la importancia de la formación en la LE y que los PNI son mejores en áreas de pronunciación: "aunque quizás no sepamos tanto vocabulario y no pronunciemos tan bien como ellos, los PNNI tenemos una carrera y una formación pedagógica; sabemos enseñar" (PNNI4, comunicación personal, 12 de mayo de 2021). Los datos de la encuesta indican un sesgo claro a favor de los PNI en áreas de pronunciación y vocabulario en línea con Lasagabaster y Sierra (2005), corroborado por los datos de los estudiantes: "en speaking y vocabulario te puede ayudar más un nativo que un profesor que solo estudió inglés y que no naciera con esa lengua" (AL6, comunicación personal, 10 de mayo de 2021), pero en contraste con Rámila (2015), ya que en este estudio prefieren la categoría de ambos profesores. Así, los PNI son caracterizados como ideales para las clases de conversación, por su mayor fluidez como en Benke y Medgyes (2005) y por la idea del acento nativo como 'modelo lingüístico': “agradezco mucho que un nativo me dé clase de inglés, ya que así mejora mi listening porque es el mismo acento que en los ejercicios" (AL1, comunicación personal, 10 de mayo de 2021), como también concluye Walkinshaw y Oahn (2014). En cuanto al área de evaluación, los datos del cuestionario no muestran una tendencia clara, en línea con Lasagabaster y Sierra (2005), aunque estos resultados contrastan con los del cuestionario, que muestran una tendencia hacia los PNNI porque les beneficia: "los nativos ven más fallos gramaticales y eso baja mucho la nota" (AL3, comunicación personal, 10 de mayo de 2021).

\section{Conclusiones}

Llegados a este punto, cabe decir que se ha contextualizado el presente trabajo dentro de la literatura actitudinal acerca del factor nativo en profesores de LE, específicamente de ILE, a partir de los estudios más relevantes, primero desde un punto de vista internacional para después centrarse en el contexto de estudio. A continuación, se articuló la metodología de investigación más adecuada para desarrollar el estudio actitudinal sobre PNI y PNI mediante un cuestionario actitudinal (LASAGABASTER; SIERRA, 2005) y grupos focales dirigidos 
tanto al alumnado como al profesorado implicado. Los resultados obtenidos revelan una tendencia positiva general de los alumnos de Bachillerato analizados hacia los PNI. Asimismo, esta preferencia hacia los PNI incrementa a medida que se completan las etapas educativas (educación primaria, secundaria y terciaria). Respecto a las preferencias del alumnado por áreas lingüísticas, los datos muestran un posicionamiento claro favorable hacia los PNI en materia de pronunciación y vocabulario, y hacia los PNNI en cuestiones relacionadas con la gramática, sobre todo. Así, surgen una serie de implicaciones pedagógicas subyacentes al estudio llevado a cabo que implican lo siguiente:

- Los alumnos se muestran a favor del acento nativo inglés, siendo para ellos el 'modelo lingüístico' (BENKE; MEDGYES, 2005), en detrimento del acento nonativo, que lo consideran incorrecto $y$, por consiguiente, muestran prejuicios hacia los PNNI, refiriéndose a ellos como inferiores (LLURDA, 2004). Debido al creciente interés del inglés como lengua franca (LLURDA; BAYYURT; SIFAKIS, 2017), es de vital importancia que los alumnos conozcan la realidad sociolingüística del inglés y sus variedades. Por tanto, existe la necesidad que el sistema educativo fomente una actitud cosmopolita del alumnado, para favorecer la cohesión social y la coexistencia de las diferentes lenguas e identidades de la comunidad (LASAGABASTER, 2017). Debe poder incluirse el componente actitudinal en el currículo, sobre todo en las asignaturas de lengua, para poder desmitificar esos prejuicios y potenciar la enseñanza del idioma. Esto se puede realizar mediante la incorporación en las programaciones de actividades reflexivas y programas de conciencia lingüística (p. ej.: debates), en los que los alumnos pudieran reflexionar tanto sobre la realidad lingüística del territorio como sobre sus propios antecedentes lingüísticos, teniendo como objetivo la aceptación de todas las diferentes opciones y variedades lingüísticas.

- A pesar de que los alumnos participantes en este estudio llevan estudiando ILE por más de una década en contextos de educación reglada, siguen mostrando problemas a la hora de aprender la lengua, e incluso señalan que tienen un nivel bajo de ILE, como se demuestra en Dewaele y Pena (2018). Igualmente, los resultados reflejan una preferencia hacia la docencia en L1, en lugar de la lengua meta, resultado de las malas prácticas docentes durante años (GARCÍA MATA, 2008) (p. ej.: la aplicación del método de gramática-traducción, donde las clases de inglés se imparten en la L1 del estudiante). En consecuencia, resulta imperativo explorar 
metodologías innovadoras para elevar la motivación de los estudiantes (LASAGABASTER, 2017) y poder paliar estos problemas.

Finalmente, se pueden enumerar varias limitaciones de esta investigación. Primero, las actitudes lingüísticas se entienden como tendencias o evaluaciones no estáticas y dinámicas, que se encuentran en un cambio constante (MCKENZIE, 2010). Resulta consecuente observarlas desde una perspectiva longitudinal y transversal para tener una visión más precisa de la realidad. Debido al constante cambio legislativo, las actitudes lingüísticas hacia la LE son más propensas a cambios, sobre todo en contextos multilingües como Galicia, donde hay varias lenguas en continuo contacto. No obstante, debido a las limitaciones de tiempo para la realización de la presenten investigación debido a la situación derivada de la COVID-19 y las medidas adoptadas por los centros educativos, se decidió desestimar la propuesta de realización de un estudio longitudinal. Además, debido a la homogeneidad de la muestra poblacional, esta impedía su división en grupos para aplicar variables como la edad o experiencia previa con PNI por parte de los participantes. Entonces, una mayor variedad de participantes permitiría estudiar diferentes variables como las mencionadas, pudiendo aportar un análisis actitudinal alternativo en cuanto al factor nativo en lo que la enseñanza de lenguas compete. Por último, aunque los resultados sean alentadores, deberían ser validados por una muestra más amplia, ya que, a veces, muestras reducidas puede que no sean representativas de todo el contexto e impiden el análisis inferencial de los datos. Aunque varios colegios fueron contactados, debido a la situación sanitaria, la mayoría decidió no participar, alegando protocolos sanitarios internos. Por último, en etapas tan exigentes como Bachillerato, los centros educativos son reticentes a permitir desarrollar investigaciones, ya que no quieren que el alumnado se distraiga de cara a la preparación de las pruebas de acceso a estudios superiores.

Respecto a posibles aplicaciones del estudio, un trabajo futuro podría implicar la utilización de observaciones de aula como instrumento complementario, como en Árva y Medgyes (2000). Según Moussu y Llurda (2008), observar las relaciones de cada alumno de manera individual junto a PNI y PNNI en el contexto educativo puede aportar una percepción más extensa de la realidad. Otra línea de investigación podría implicar la exploración de la opción de ambos profesores, incluyéndolo en las diferentes categorías del cuestionario actitudinal, ya que algunos autores recuerdan su importancia a la hora de entender las preferencias de los alumnos ante PNI y PNNI (LASAGABASTER; SIERRA, 2005; MEDGYES, 1992; RÁMILA, 2015). Al mismo tiempo, otra vía inexplorada es el análisis de las percepciones de PNI y PNNI, teniendo en cuenta variables como los años de experiencia 
docente o su grado de exposición al inglés mediante estancias en países de habla inglesa, por ejemplo, siendo una variable diferencial como mencionan Llurda y Huguet (2003). De igual forma, también cabe la posibilidad de tener en cuenta otras variables como la edad o el género, como hacen Lasagabaster y Sierra (2002), aunque no sería relevante en cuanto al contexto educacional del presente trabajo debido a la homogeneidad de la muestra. Asimismo, otra opción supondría el análisis de los datos del cuestionario relativos a la preferencia en áreas lingüísticas específicas según la etapa educativa como en Lasagabaster y Sierra (2005). Por último, con una muestra más amplia, sería interesante investigar la autoconciencia de nivel en la LE de los estudiantes, correlacionado con las actitudes hacia los PNI y PNNI, tal y como hacen Dewaele y Pena (2018). Aunque esta variable fue analizada aquí, el análisis inferencial de esta variable no fue incluida finalmente debido a su no significancia por la limitación de la muestra de población tratada.

\section{Referencias}

ALONSO-HERRERO, Ane; LASAGABASTER, David. Student attitudes towards English pronunciation and different varieties in the English classroom. Estudios de Linguiística Inglesa Aplicada, 19, p. 71-100. 2019.

ANDREWS, Stephen. Teacher language awareness. Cambridge: Cambridge University Press, 2007.

ÁRVA, Valeria; MEDGYES, Péter. Native and non-native teachers in the classroom. System, 28-3, p. 355-372. 2000.

BAKER, Colin. Attitudes and language. Clevedon: Multilingual Matters, 1992.

BENKE, Eszter; MEDGYES, Péter. Differences in teaching behaviour between native and nonnative speaker teachers: As seen by the learners. En LLURDA, Enric. Non-Native Language Teachers: Perceptions, Challenges, and Contributions to the Profession, p. 195-215. Nueva York: Springer, 2005.

BRAINE, George Non-native educators in English language teaching. Mahwah, NJ: Lawrence Erlbaum Associates, 1999.

BRUTT-GRIFFLER, Janina; SAMIMY, Keiko. Transcending the nativeness paradigm. World Englishes, 20-1, p. 99-106, 2001.

CALAFATO, Raees. The non-native speaker teacher as proficient multilingual: A critical review of research from 2009-2018. Lingua, 227, p. 1-25, 2019.

CARLESS, David, y WALKER, Elizabeth. Effective team teaching between local and nativespeaking English teachers. Language and Education, 20-6, p. 463-477, 2006.

DEWAELE, Jean-Marc. Why the dichotomy 'L1 versus LX user' is better than 'native versus non-native speaker'. Applied Linguistics, 39-2, p. 236-240, 2018.

DEWAELE, Jean-Marc; PENA, Carmen. Sources of variation in Galician multilinguals' attitudes towards Galician, Spanish, English, and French. Revista Nebrija de Lingüística Aplicada a la Enseñanza de las Lenguas, 15-25, p. 34-58, 2018.

DOR̈NYEI, Zoltán; CSIZER, Kata; NEMETH, Nãra. Motivation, Language, Attitudes and Globalisation. Clevedon: Multilingual Matters, 2006.

EAGLY, Alice; CHAIKEN, Shelly. The Psychology of Attitudes. Fort Worth, TX: Harcourt Brace Jovanovich College Publishers, 1993. 
EBERHARD, David; SIMONS, Gary; FENNIG, Charles. Ethnologue: Languages of the world, twenty-third edition, 2021. Disponible en: https://www.ethnologue.com/language/eng. Acceso el: 11 de octubre de 2021.

EDUCATION FIRST. EF EPI 2020: Índice EF de nivel de inglés. Educational First Publishing, 2020. Dispoible en: https://www.ef.com.es/epi/. Acceso el: 11 de octubre de 2021. EDWARDS, John. Multilingualism. Londres y Nueva York: Routledge, 1994.

FAUZI, Nabilah; HASHIM, Harwati. Apple vs. Mangosteen: A qualitative study of students' perception towards native and non-native English-speaking teachers. Journal of Education and E-Learning Research, 7-2, p. 218-228, 2020.

GARCÍA MATA, Jorge. Algunas causas de la mala práctica docente en lenguas extranjeras. Porta Linguarum, 10, p. 101-114, 2008.

GARRETT, Peter. Attitudes to Language. Cambridge: Cambridge University Press, 2010. GIBSON, Barbara; HUA, Zhu. (2016). Interviews. En HUA, Zhu. Research Methods in Intercultural Communication: A Practical Guide, p. 180-195. Malden, MA: Wiley Blackwell, 2016.

HOLLIDAY, Adrian (2015) Native-speakerism: Taking the concept forward and achieving cultural belief. En: SWAN, Anne; ABOSHIHA, Pamela; HOLLIDAY, Adrian. (En)Countering Native-speakerism. Palgrave Advances in Language and Linguistics. Londres: Palgrave Macmillan, 2015.

JENKINS, Jennifer. Exploring attitudes towards English as a lingua franca in the East Asian context. En: MURATA, Kumiko; JENKINS, Jennifer. Global Englishes in Asian Contexts: Current and Future Debates, p. 40-56. Houndmills: Palgrave Macmillan, 2009.

JENKINS, Jennifer. Repositioning English and multilingualism in English as a lingua franca. Englishes in Practice, 2-3, p. 49-85, 2015.

JOHNSTONE, Tony. Questionnaires and surveys. En: HUA, Zhu. Research Methods in Intercultural Communication: A Practical Guide, p. 165-179. Malden, MA: Wiley Blackwell, 2016.

KACHRU, Braj. The Indianness in Indian English. Word, 21, p. 391-410, 1965.

KACHRU, Braj. Standards, codification, and sociolinguistic realism: The English language in the outer circle. En: QUIRK, Randolph; WIDDOWSON, Henry. English in the World: Teaching and Learning the Language and Literatures. p. 11-31. Cambridge: Cambridge University Press, 1985.

LAI PING, Florence. Advantages and disadvantages of native- and nonnative-English-speaking teachers: Student perceptions in Hong Kong. TESOL Quarterly, 46-2, p. 280305, 2012.

LASAGABASTER, David.; HUGUET, Angel. Multilingualism in European bilingual contexts: Language use and attitudes. Clevedon: Multilingual Matters, 2007.

LASAGABASTER, David. Language learning motivation and language attitudes in multilingual Spain from an international perspective. The Modern Language Journal, 101-3, p. 583-596, 2017

LASAGABASTER, David; SIERRA, Juan Manuel. University students' perceptions of native and non-native speaker teachers of English. Language Awareness, 11-2, p. 132-142, 2002.

LASAGABASTER, David; SIERRA, Juan Manuel. What do students think about the pros and cons of having a native speaker teacher? En: LLURDA, Enric. Non-native Language Teachers: Perceptions. Challenges and Contributions to the Profession, p. 217-241. Boston, MA: Springer, 2005.

LLURDA, Enric. Non-native-speaker teachers and English as an international language. International Journal of Applied Linguistics, 14-3, p. 314-323, 2004.

LLURDA, Enric. Non-native TESOL students as seen by practicum supervisors. En LLURDA, 
Enric. Non-native Language Teachers: Perceptions, Challenges, and Contributions to the Profession, p. 131-154. Boston, MA: Springer, 2005.

LLURDA, Enric. Methods in NNEST research. The TESOL Encyclopedia of English Language Teaching, 1, p. 1-6, 2018.

LLURDA, Enric; HUGUET, Angel. Self-awareness in NNS EFL primary and secondary school Teachers. Language Awareness, 12-4, p. 220-233, 2003.

LLURDA, Enric; BAYYURT, Yasemin; SIFAKIS, Nikos. Raising teachers' awareness about English and English as a lingua franca. En: GARRET, Peter; COTS, Josep. The Routledge Handbook of Language Awareness, p. 155-169. Londres: Routledge, 2017.

MADRID, Daniel; PÉREZ CAÑADO, María Luisa. Teacher and student preferences of native and non-native foreign language teachers. Porta Linguarum, 2, p. 125-138, 2004.

MANSFIELD, Gillian; POPPI, Franca. The English as a foreign language/lingua franca debate: Sensitizing teachers of English as a foreign language towards teaching English as a lingua franca. Profile, 14-1, p. 159-172, 2012.

MCKENZIE, Robert. The Social Psychology of English as a Global Language. Attitudes, Awareness, and Identity in the Japanese Context. Londres: Springer, 2010.

MEDGYES, Péter. Native or non-native: who's worth more? ELT Journal, 46-4, p. 340-349, 1992.

MOUSSU, Lucie; LLURDA, Enric. Non-native English-speaking English language teachers: History and research. Language Teaching, 41-3, p. 315-348, 2008.

O'ROURKE, Bernadette. Galician and Irish in the European Context: Attitudes towards Weak and Strong Minority Languages. Houndmills y Nueva York: Palgrave Macmillan, 2011. PÉREZ VIDAL, Carmen. Language acquisition in three different contexts of learning: Formal instruction, study abroad, and semi-immersion (CLIL). En: GALLARDO DEL PUERTO, Francisco; RUIZ DE ZAROBE, Yolanda; SIERRA, Juan Manuel. Content and foreign language integrated learning: Contributions to multilingualism in European contexts, p. 103127. Berna y Nueva York: Peter Lang, 2011.

RÁMILA, Noemí. Students' preferences regarding native and non-native teachers of English at a University in the French Brittany. Procedia, Social and Behavioral Sciences, 173, p. 9397, 2015.

REAL DECRETO 1105/2014, de 26 de diciembre, por el que se establece el currículo básico de la Educación Secundaria Obligatoria y del Bachillerato (BOE $n^{\circ}$ 3, 3 de enero de 2015).

REVES, T.; MEDGYES, Péter. The non-native English speaking EFL/ESL teacher's selfimage: An international survey. System, 22-3, p. 353-367, 1994.

SELVI, Ali. All teachers are equal, but some teachers are more equal than others: Trend analysis of job advertisements in English language teaching. WATESOL NNEST Caucus Annual Review, 1, p. 156-181, 2010.

SU, Wei. Two heads better than one? Exploring the co-teaching of intercultural competence by NES and NNS teachers. The Asia-Pacific Education Researcher, 30-2, p. 95-108, 2021.

SU, Wei; SHANG, Xiaoqi. NNS and NES teachers' co-teaching of interpretation class: A case study. The Asia-Pacific Education Researcher, 29-4, p. 353-364, 2020.

TODD, Watson; POJANAPUNYA, Punjaporn. Implicit attitudes towards native and nonnative speaker teachers. System, 37-1, p. 23-33, 2009.

TSOU, Wenli; CHEN, Fay. EFL and ELF college students' perceptions toward Englishes. Journal of English as a Lingua Franca, 3-2, p. 363-386, 2017.

VILLACAÑAS, Luis. Panorama de la enseñanza de lenguas extranjeras en la España moderna. En: BARBERÁ, Óscar; MAYORDOMO, Alejndro. Escoles i Mestres. Dos Siglos de Historia y Memoria en Valencia, p. 382-401. Universidad de Valencia, Valencia, 2017.

WALKINSHAW, Ian; OANH, Duongthi. Native and non-native English language teachers. Sage Open, 4-2, p. 1-9, 2014. 
ZHANG, Yangxian. Reviewing non-native English-speaking teachers' professional identity. International Journal of Languages Education and Teaching, 4-3, p. 320-335, 2016.

\section{SOBRE O/AS AUTOR/AS}

Iván Martínez Rivas. Máster en Formación del Profesorado de Educación Secundaria Obligatoria y Bachillerato, Formación Profesional y Enseñanzas de Idiomas (2021), Universidad Alfonso X el Sabio (UAX)- España.

José Luis Estrada Chichón. Doctor en didáctica de lenguas extranjeras (2017), Universidad de Cádiz (UCA), España; profesor del Departamento de Didáctica de la Lengua y la Literatura de la UCA-España; miembro del grupo de investigación HUM-485 Enseñanza de lenguas extranjeras: Materiales para un nuevo diseño curricular (UCA).

\section{Como citar este artigo (ABNT):}

RIVAS, Iván Martínez; CHICHÓN, José Luis Estrada. Actitudes lingüísticas hacia el profesorado de inglés nativo y no nativo. Revista Práxis Educacional, Vitória da Conquista, v. 18, n. 49, 2022. https://doi.org/10.22481/praxisedu.v18i49.9639 